\title{
Instrumento para avaliação de detergentes enzimáticos
}

\author{
AN INSTRUMENT FOR EVALUATING ENZYMATICS DETERGENTS
}

INSTRUMENTO PARA EVALUACIÓN DE DETERGENTES ENZIMÁTICOS

\author{
Denise Rodrigues Costa Schmidt ${ }^{1}$, Christiane Sayuri Ito Yonekura ${ }^{2}$, Rosineide Feres Gil ${ }^{3}$
}

\section{RESUMO}

Estudo de desenvolvimento metodológico que teve por objetivo construir um instrumento de avaliação de detergentes enzimáticos e analisar a ação dos produtos sobre os instrumentais. 0 instrumento foi submetido à análise de três juízes. 0 préteste foi realizado com 25 instrumentos. A amostra para a análise de campo foi constituída por mais 25 instrumentais cirúrgicos. Os resultados obtidos permitiram a construção de um instrumento com 19 itens e a análise de campo constatou que houve diferenças nos escores das soluções avaliadas. 0 produto $\mathrm{P} 5$ obteve a melhor média 78,7 (DP 6,68) e mediana 80,2 e o produto P4 obteve o pior desempenho, média 56,2 (DP 7,09) e mediana 56,7, em um intervalo possível de 19 a 95 . 0 instrumento de medida mostrou-se confiável ao estudo, apresentando valor do Alpha de Cronbach de 0,83 .

\section{DESCRITORES}

Detergentes.

Instrumentos cirúrgicos.

Esterilização.

\begin{abstract}
The objective of this study was to develop an instrument for measuring the quality of enzymatic detergents and analyze their cleaning action on surgical instruments. The instrument was submitted to a panel of three judges. It was pre-tested in a sample of 25 surgical instruments. Twentyfive other surgical instruments trays were used for the field analysis. The results made possible the construction of an instrument with 19 items, and the field analysis verified that there were differences in the scores of the solutions that were evaluated. P5 had the best average score, 78.7 (SD 6.68), and median of 80.2 , and P4 had the worst performance, with an average score of 56.2 (SD 7.09) and median of 56.7 in a possible range between 19 and 95 . In the study, the measuring instrument proved to be reliable, with a Cronbach's alpha of 0.83 .
\end{abstract}

\section{KEY WORDS}

Detergents.

Surgical instruments.

Sterilization.

\section{RESUMEN}

Se trata de un estudio de desarrollo metodológico que tuvo como objetivo construir un instrumento de evaluación de detergentes enzimáticos y analizar la acción de los productos sobre los instrumentales. El instrumento fue sometido al análisis de tres jueces. El pre test fue realizado con 25 instrumentos. La muestra para el análisis de campo estuvo constituida por más de 25 instrumentales quirúrgicos. Los resultados obtenidos permitieron la construcción de un instrumento con 19 items y por el análisis de campo se constató que hubo diferencias en los escores de las soluciones evaluadas. El producto $\mathrm{P} 5$ obtuvo el mejor promedio de 78,7 (DP 6,68) y mediana de 80,2 y el producto $P 4$ obtuvo el peor desempeño, promedio de $56,2($ DP 7,09$)$ y mediana de 56,7 , en un intervalo posible de 19 a 95 . El instrumento de medida se mostró confiable para el estudio, presentando valor del Alpha de Cronbach de 0,83.

\section{DESCRIPTORES}

Detergentes.

Instrumentos quirúrgicos.

Esterilización.

\footnotetext{
${ }^{1}$ Enfermeira Centro Cirúrgico Hospital Universitário de Londrina-Paraná, doutoranda da Escola de Enfermagem de Ribeirão Preto, USP. Londrina, PR, Brasil. denisebeto@terra.com.br ${ }^{2}$ Enfermeira da Central de Materiais e Esterilização do Hospital Universitário de Londrina - Paraná. Especialista em Centro Cirúrgico, Central de Materiais e Recuperação Anestésica - Universidade Estadual de Londrina. Londrina, PR, Brasil. ${ }^{3}$ Enfermeira da Central de Materiais e Esterilização do Hospital Universitário de Londrina - Paraná. Especialista em Centro Cirúrgico, Central de Materiais e Recuperação Anestésica Universidade Estadual de Londrina. Londrina, PR, Brasil.
} 


\section{INTRODUÇÃO}

A Central de Materiais e Esterilização (CME) é uma unidade de apoio técnico a todas as unidades assistenciais, responsabilizando-se pelo processamento dos artigos, desde a limpeza, preparo, esterilização e distribuição às unidades consumidoras ${ }^{(1)}$.

Nesta unidade 0 avanço tecnológico tem sido uma constante, principalmente após a década de $70^{(2)}$, ocorrendo, inclusive um aprimoramento nos processos de limpeza. 0 processo de limpeza visa a remoção da sujidade visível e, por conseqüência, a retirada da carga microbiana inicial em $99,9 \%$, ou seja, redução de quatro ciclos logarítimicos do bioburden presente no artigo ${ }^{(3)}$. Esse processo é indispensável para o reprocessamento de todos os artigos médico-hospitalares e deve preceder os processos de desinfecção e esterilização.

As técnicas de limpeza têm se tornado bastante consistentes, reforçando a mesma como passo inicial e fundamental para garantir as fases posteriores ${ }^{(4)}$. A prática de enfer-magem na CME é bastante complexa devido à diversidade de atividades setoriais. Por suas características peculiares, essas atividades, como a limpeza dos artigos médico-hospitalares, exigem conhecimento específico e diversificado, que podem ser adquiridos de modo formal ou informal(5).

$\mathrm{Na}$ atualidade, procedimentos diversificados para a limpeza de artigos têm sido utilizados em larga escala. Alguns desses procedimentos incluem o uso de produtos denominados limpadores ou detergentes enzimáticos, que por meio de seus compostos multi-enzimáticos, surfactantes, solubilizantes e álcool isopropílico têm como objetivos proporcionar um processo de limpeza rápido, eficaz, que garanta a maior conservação dos artigos médico-hospitalares e a maior segurança dos profissionais da saúde, por meio da minimização da exposição dos mesmos aos riscos biológicos ${ }^{(4,6)}$.

A utilização destes produtos juntamente com a limpeza mecânica na rotina diária da CME pode proporcionar, também, a redução da sobrecarga de trabalho dos técnicos/ auxiliares de enfermagem desta unidade.

0 que pode ser observado no desenvolvimento desta rotina na CME, é o surgimento de um número excessivo de detergentes enzimáticos, com marcas e características distintas, dificultando a opção e a utilização dos mesmos ${ }^{(7)}$.

As dificuldades encontradas dizem respeito, principalmente, às características de diluição dos produtos, 0 que pode dificultar o treinamento dos servidores envolvidos neste processo, e ao processo licitatório, no caso das instituições públicas.
Assim, surgiu o interesse em disponibilizar um instrumento que auxiliasse 0 enfermeiro da CME durante a análise dos detergentes enzimáticos no processo de limpeza manual, auxiliando-o no processo de compra deste produto.

Na revisão da literatura realizada constatou-se uma deficiência de estudos que abordem exclusivamente este tema $a^{(6-8)}$. Os estudos encontrados valorizaram a esterilização e a validação da mesma ${ }^{(9-11)}$, o reprocessamento de artigos de uso único ${ }^{(3,12)}$ e a validação das lavadoras desinfectoras ${ }^{(4)}$, não apresentando uma abordagem sobre a qualidade dos detergentes enzimáticos.

\section{OBJETIVOS}

1. Propor um instrumento para avaliação de detergentes enzimáticos;

2. Avaliar, por meio da aplicação do instrumento, o resultado da limpeza de artigos médico-hospitalares utilizando cinco marcas de detergentes enzimáticos distintas. que pode ser observado é o surgimento de de detergentes enzimáticos, com marcas e características distintas, dificultando a utilização mesmos.

\section{MÉTODO}

Trata-se de um estudo de desenvolvimento metodológico, realizado na CM E de um hospital escola da cidade de Londrina Paraná. Após estabelecer a teoria sobre o construto para o qual se destinava desenvolver 0 instrumento, foi realizada uma revisão da literatura, a qual auxiliou na composição e disposição dos itens do instrumento.

0 primeiro instrumento elaborado foi, então, composto por 20 itens, dispostos em uma escala ordinal do tipo Likert de cinco pontos, varia-ndo de 1 (nenhuma/nada) a 5 (totalmente), 0 qual foi submetido à validação de conteúdo.

Para a validação de conteúdo foram escolhidos três juízes, os quais tiveram o objetivo de refinar a redação dos itens e avaliar a pertinência conceitual, assim como realizar a análise semântica da primeira versão do instrumento. Os juízes foram enfermeiros com conhecimento sobre a temática, experiência no ensino ou na prática gerencial de enfermagem e que concordaram em participar do estudo.

Os três juízes foram convidados a participar do estudo mediante contato telefônico, quando foram expostos os objetivos do estudo e solicitada a sua participação. Diante da aceitação de todos, foi enviado aos juizes um instrumento o qual continha os três fatores, as suas respectivas definições constitutivas e outra tabela de dupla entrada com os fatores e os itens. Com base nesta tabela, a função dos juízes consistiu em colocar um $X$ para $o$ item debaixo do fator ao qual o juiz julgou $o$ item se referir ${ }^{(13)}$. 
Após os ajustes decorrentes do julgamento dos juizes, o instrumento adquiriu uma nova formatação, com 22 itens e três domínios. Desta forma, foi realizado o préteste com o novo instrumento para a análise das dificuldades de administração, interpretação e concepção do sistema de pontuação. O sistema adotado para a atribuição dos escores partiu de um modelo(14), no qual algumas frases são consideradas negativas e outras positivas, o que significa que os escores das frases com direcionamento positivo devem ser invertidos, ou seja, possuem escores reversos. No modelo adotado para este estudo ${ }^{(14)}$, a direção geral da escala é positiva, o que significa que quanto maior o escore melhor a qualidade do produto.

0 pré-teste foi realizado pelas autoras do estudo, utilizando a observação do material e da solução enzimática, a olho nu e por meio de lupa após o processo de limpeza. A amostra do pré-teste foi constituída por 25 caixas de instrumentais provenientes do centro cirúrgico, dentre as quais foram sorteados os instrumentos para cada tipo de solução, totalizando cinco instrumentos para cada solução.

Os dados obtidos decorrentes da realização do préteste foram submetidos à análise estatística, 0 que acarretou na exclusão de três itens, os quais obtiveram valores do Alpha de Cronbach inferior a 0,50.

0 instrumento final foi composto, portanto, por questões relacionadas às características do produto, características do instrumental e em relação à presença de sangue na superfície dos artigos e pelos 19 itens relacionados à adequação da embalagem, adequação do produto e ação do produto sobre o instrumental (apêndice), mantendo-se as opções de resposta em escala de Likert de cinco pontos. Assim, o intervalo possível de pontuação do instrumento variou de 19 a 95, o que significa que quanto maior o escore, melhor a qualidade do detergente enzimático.

Para a análise do escore total do instrumento as autoras utilizaram a soma de todos os escores atribuídos a cada um dos 19 itens que compõem o instrumento. Antes, porém, da realização deste cálculo, foi necessária a inversão dos escores dos itens $1 ; 2 ; 9 ; 10 ; 12 ; 13 ; 14 ; 16 ; 18$; 19 , os quais possuem escores reversos ${ }^{(14)}$.

Para facilitar a interpretação dos resultados, utilizamos o cálculo do escore médio, o qual foi obtido por meio da divisão entre o escore total e o número de itens do instrumento (19). 0 resultado foi interpretado em um intervalo possível de 1 a 5 , no qual $1=$ péssimo; $2=$ regular; $3=$ nem bom/ nem ruim; $4=$ muito bom e $5=$ excelente $^{(14)}$. Por exemplo, se o produto avaliado obtiver um escore total igual a 57, este valor será dividido pelo número de itens (19), obtendo-se assim um valor para o escore médio igual a 3. Esse valor obtido é interpretado na escala proposta, de 1 a 5 , obtendo o conceito médio, ou seja, nem bom/nem ruim.

Pode-se calcular o escore para cada um dos três componentes do instrumento, o qual é efetuado pela somatória dos escores atribuídos para cada componente separadamente. Devido ao número distinto de itens em cada etapa, utiliza-se a razão entre os escores de cada componente e o número de itens que compõe cada fase, conforme demonstrado no Quadro 1.

Quadro 1 - Itens de cada componente, intervalo de medidas - Londrina - 2006

\begin{tabular}{|c|c|c|c|}
\hline Componentes & $\begin{array}{l}\text { Itens negativos }(1=1 ; 2=2 ; \\
3=3 ; 4=4 \text { e } 5=5)\end{array}$ & $\begin{array}{l}\text { Itens positivos }(1=5 ; 2=4 ; 3=3 ; \\
4=2 ; 5=1)\end{array}$ & Escores possíveis \\
\hline Adequação da Embalagem & $3 ; 4 ; 5 ; 8$ & - & $4-20(1-5)$ \\
\hline Características do Produto & $6 ; 7 ; 17$ & $1 ; 2$ & $5-25(1-5)$ \\
\hline Ação do Produto & $11 ; 15$ & $9 ; 10 ; 12 ; 13 ; 14 ; 16 ; 18 ; 19$ & $10-50(1-5)$ \\
\hline
\end{tabular}

Após a realização do pré-teste e adequação do sistema de escore foi realizado o teste em campo para verificar se 0 instrumento aplica-se ao que foi proposto. A coleta de dados também foi realizada pelas autoras do estudo, nos meses de junho e julho de 2006, por meio da aplicação do instrumento de avaliação de detergente enzimático em uma amostra de 55 instrumentos, totalizando 11 instrumentos para cada solução enzimática.

0 procedimento de análise dos resultados foi obtido por meio da observação do material e da solução enzimática, a olho nu e por meio de lupa após o processo de limpeza. Os instrumentos foram imersos separadamente em recipientes contendo o produto enzimático imediatamente após a sua chegada ao expurgo da CME, não per- manecendo parados após o uso. A cada análise realizada, a solução enzimática foi trocada, uma vez que a presença de matéria orgânica na solução poderia comprometer a realização do estudo.

A avaliação da quantidade de sangue visível na superfície dos artigos, foi realizada subjetivamente, uma vez não havia como medir a quantidade exata de sujidade presente nos instrumentos. Optou-se, no entanto, que essa análise fosse realizada de forma concomitante pelas autoras, ocorrendo a avaliação de outra pessoa quando da ocorrência de discordância entre as autoras.

Os detergentes enzimáticos que fizeram parte do estudo foram compostos multi-enzimáticos, com pH neutro, 
biodegradáveis e possuir autorização de comercialização pelo Ministério da Saúde e notificado na Agência Nacional de Vigilância Sanitária (ANVISA). Os mesmos foram identificados como P1 (Produto 1), P2 (Produto 2), P3 (Produto 3), P4 (Produto 4) e P5 (Produto 5).

Para o tratamento dos dados perdidos (missing data), no caso em que não houve o preenchimento completo da escala, seguiu-se o critério apresentado na literatura ${ }^{(15)}$, 0 qual estabelece que só deva ser excluídos da amostra aqueles instrumentos que tiveram $20 \%$ ou mais de itens não respondidos.

Os dados obtidos por meio da coleta de dados foram digitados no programa estatístico Statistical Package for the Social Science version 11.5 for W indows (SPSS), analisados por meio de estatística descritiva e apresentados em tabelas e gráficos tipo Boxplots. Para análise comparativa das diferenças encontradas entre os escores dos detergentes enzimáticos, utilizou-se o teste não paramétrico de Kruskal Wallis (nível de significância de 0,05).

A consistência interna do instrumento foi obtida pelo cálculo do Alpha de Cronbach,verificando a congruência que cada item do teste tem com o restante dos itens do mesmo teste ${ }^{(13)}$.

\section{RESULTADOS}

Na análise da consistência interna, pelo Alfa de Cronbach, observamos que o valor de Alfa manteve-se elevado $(0,83)$, o que indica a alta consistência interna do instrumento. Como critério de exclusão dos itens do primeiro instrumento, adotou-se o valor de 0,50 , bem como a verificação da natureza do item e da influência da sua exclusão no valor do Alfa.

A análise de conteúdo, realizada por juízes, verificou a adequação da representação do atributo e completou os procedimentos teóricos da construção do instrumento, mostrando-se adequada. No que se refere ao formato do instrumento, optou-se pela escala do tipo Likert por ser um dos modelos mais utilizados ${ }^{(13)}$.

Para a permanência de um item após a análise dos juízes é necessária a concordância de pelo menos $80 \%$ dos mesmos ${ }^{(13)}$. Neste estudo aceitou-se como critério a concordância de dois dos três juízes. Este índice de concordância foi obtido pela maioria dos itens.

0 sistema de pontuação foi adequado para a realização do estudo, mostrando-se também de fácil aplicação.

Os resultados obtidos demonstraram que $49,1 \%$ dos instrumentais continham média quantidade de sujidade e $94,5 \%$ apresentaram sangue em sua superfície. 0 maior número de artigos presentes neste estudo foram os utilizados nas cirurgias ginecológicas $(38,2 \%)$. A diluição dos produtos variou, dependendo do fabricante, de dois a quatro mililitros de solução enzimáti$\mathrm{ca/litro} \mathrm{de} \mathrm{água;} \mathrm{sendo} \mathrm{que} \mathrm{somente} \mathrm{os} \mathrm{produtos} \mathrm{iden-}$ tificados como P1 e P3 indicavam diluição de quatro mililitros de solução por litro de água. 0 tempo de contato com o instrumental variou de dois a cinco minutos e apenas o produto P4 indicava tempo de contato de cinco minutos.

0 número de enzimas nos produtos analisados variou de três a quatro enzimas, porém, somente o produto $\mathrm{P} 3$ utilizava três enzimas em sua composição e o produto P4 não informava este dado em seu rótulo. Consideramos informações essenciais como o tempo de contato, diluição e a composição da solução são fundamentais para garantir a qualidade de um produto.

0 resultado referente à avaliação das cinco marcas de detergentes enzimáticos utilizadas neste estudo, por meio do instrumento de avaliação, está apresentado na Tabela 1.

Tabela 1 - Estatística descritiva da avaliação dos detergentes enzimáticos em uma CME do município de Londrina Londrina - 2006

\begin{tabular}{cccccc}
\hline Produto & $\begin{array}{c}\text { Intervalo } \\
\text { Possível }\end{array}$ & $\begin{array}{c}\text { Intervalo } \\
\text { Obtido }\end{array}$ & Mediana & Média Escore Total & $\begin{array}{c}\text { Desvio } \\
\text { padrão }\end{array}$ \\
\hline P1 & $19-95$ & $69-81$ & 76,3 & 76,4 & 3,82 \\
P2 & $19-95$ & $57-73$ & 63,2 & 63,5 & 4,72 \\
P3 & $19-95$ & $57-82$ & 76,3 & 74,5 & 6,66 \\
P4 & $19-95$ & $43-65$ & 56,7 & 56,2 & 7,09 \\
P5 & $19-95$ & $63-86$ & 80,2 & 78,7 & 6,68 \\
\hline
\end{tabular}

Na Tabela 1 observa-se que o produto P5 obteve a meIhor média para o Escore Total do instrumento, 78,7 (DP6,68) e mediana 80,2 e o produto P4 obteve o pior desempenho, média 56,2 (DP7,09) e mediana 56,7, em um range possível de 19 a 95. De acordo com a pontuação estabelecida pelas autoras para este estudo, o produto
P5 apresentou a melhor qualidade e o produto P4 a pior qualidade.

Na Figura 1 encontra-se a distribuição das medianas dos produtos, cuja diferença apresentou-se estatisticamente significante $(p<0,05)$. 


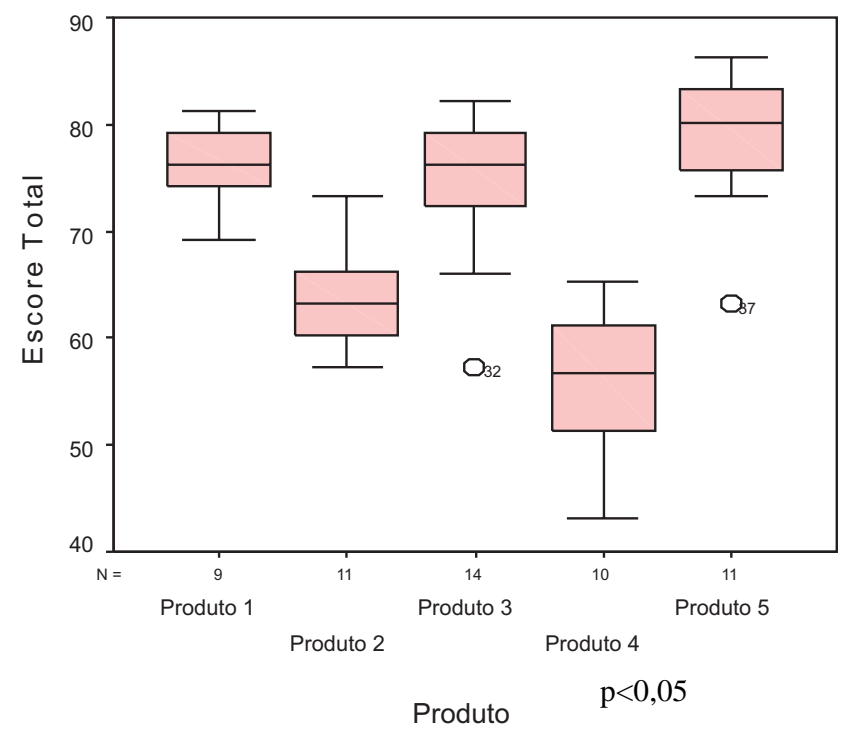

Figura 1 - Distribuição das medianas dos produtos analisados segundo o valor do Escore Total do instrumento Londrina - 2006

Tabela 2 - Estatística descritiva da avaliação dos escores médios dos detergentes enzimáticos em uma CME do município de Londrina - Londrina - 2006

\begin{tabular}{ccccc}
\hline Produto & $\begin{array}{c}\text { Intervalo } \\
\text { Possível }\end{array}$ & Mediana & $\begin{array}{c}\text { Média Escore } \\
\text { Total }\end{array}$ & Desvio padrão \\
\hline P1 & $19-95$ & 4,3 & 4,3 & 0,1 \\
P2 & $19-95$ & 3,7 & 3,8 & 0,2 \\
P3 & $19-95$ & 4,3 & 4,2 & 0,3 \\
P4 & $19-95$ & 3,4 & 3,3 & 0,3 \\
P5 & $19-95$ & 4,6 & 4,5 & 0,3 \\
\hline
\end{tabular}

Na Tabela 2 apresentamos os escores médios do instrumento.

Este resultado demonstra que o produto P5 encontrase entre muito bom e excelente, enquanto o produto $\mathrm{P} 4$ encontra-se entre nem bom/nem ruim.

$\mathrm{Na}$ análise comparativa do escore do fator relacionado à ação do produto sobre o instrumental e a quantidade de sujidade (Figura 2), verificou-se que quanto menor 0 volume de sujidade no artigo, melhor o resultado da limpeza, isto é, melhor a pontuação do produto.

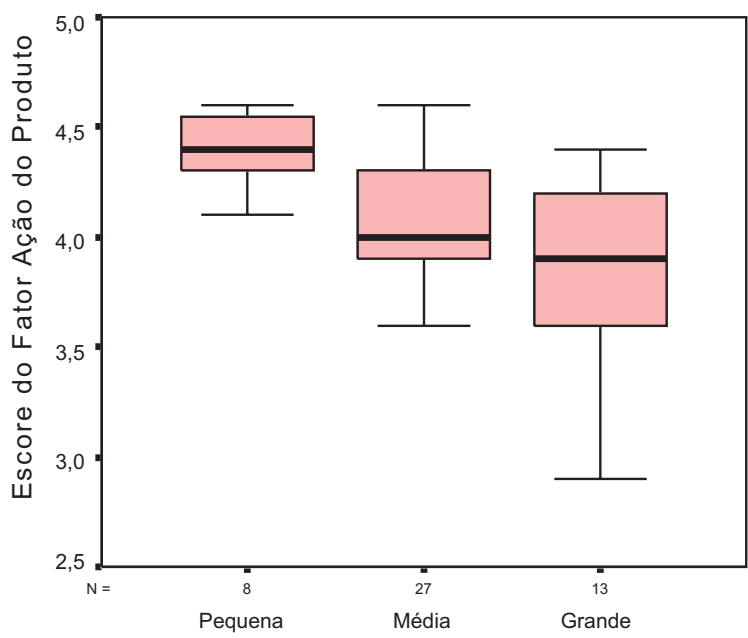

Quantidade de Sujidade

Figura 2 - Relação do escore do fator ação do produto e quantidade de sujidade - Londrina - 2006 


\section{DISCUSSÃO}

Neste estudo, o instrumento proposto foi submetido à análise de pelo comitê de juízes, cujas sugestões contribuíram para 0 aperfeiçoamento do instrumento. A etapa do pré-teste também se mostrou eficaz, no sentido de atender os objetivos de verificar a compreensão do instrumento e testar o sistema de atribuição dos escores. A análise estatística dos dados do pré-teste, embora em número insuficiente para a construção de instrumentos, atendeu ao objetivo de identificar itens redudantes.

A grande preocupação com as análises de confiabilidade e validade de escalas tem sido ratificada por alguns autores ${ }^{(16)}$, com vistas à análise da sua qualidade, e embora esta etapa não tenha sido atingida neste estudo, consideramos que 0 instrumento pode ser utilizado para verificar a qualidade dos detergentes enzimáticos encontrados no mercado nacional.

A realização da confiabilidade, feita pela análise de consistência interna apresentou o valor de Alfa total igual a 0,83 , indicando ser consistente, podendo ser utilizado no auxílio de enfermeiros no gerenciamento da CME.

No presente estudo, a maioria dos artigos médico hospitalares apresentava sangue em sua superfície. Em um estudo sobre limpeza de instrumentais foi verificado que os artigos sujos com sangue ou muco apresentavam limpeza efi-ciente após imersão em solução enzimática, porém, quando se tratava da presença de óleo ou antibiótico, os instrumentais necessitavam de limpeza manual|(6), significando que os artigos utilizados neste estudo poderiam apresentar um melhor processo de limpeza, dependendo da quantidade de matéria orgânica presente em sua superfície.

A quantidade de sujidade parece ter influenciado a ação do detergente enzimático e independente do fabricante, os resultados apresentados na Figura 2, reforçam a recomendação de necessidade de escovar o material após a imersão em solução enzimática.

A limpeza e eficiência do processo estão relacionadas também ao tempo adequado de imersão dos artigos na solução enzimática. 0 tempo de imersão sugerido pelos fabricantes dos produtos analisados foi de dois a cinco minutos. 0 curto tempo de imersão do artigo em solução enzimática pode agilizar os procedimentos de preparo e esterilização, sendo este aspecto favorável para o gerenciamento da CME. A maior agilidade no processo de limpeza pode, inclusive, facilitar a distribuição de cirurgias no centro cirúrgico devido a maior rapidez na liberação de materiais esterilizados.

O número de enzimas de um detergente enzimático também é essencial no procedimento de limpeza dos artigos médico-hospitalares. Existem milhares de enzimas diferentes e cada uma cataliza uma reação química específica(6). As enzimas, componentes dos detergentes enzimáticos, são classificadas dentro de quatro grupos maiores, como a amilase, lipase, protease e carbohidrase, obtidas por meio de fontes, como plantas, animais e microorganismose acrescidas de tenso ativo não iônico que potencializam a degradação e a remoção dos resíduos orgânicos de difícil remoção, como sangue, tecido, pus, fezes e muco ${ }^{(6-7)}$.

0 efeito obtido pelo detergente enzimático sobre o instrumental é a remoção de toda matéria orgânica visível, assim como em locais menos acessíveis, como lúmens, canais e articulações ${ }^{(7)}$.

Além disso, as fórmulas enzimáticas propiciam um processo que minimiza os riscos encontrados no ambiente de trabalho, pela significante redução, senão eliminação da ne-cessidade de manuseio de instrumentais contaminados ${ }^{(6-7)}$.

Neste estudo, os cinco produtos analisados apresentaram escores distintos com a aplicação do instrumento proposto. Houve diferença estatisticamente significante entre os escores, sendo que o produto P5 apresentou meIhor qualidade em relação aos demais detergentes. Este resultado sugere que os detergentes enzimáticos necessitam de uma avaliação criteriosa quanto a sua qualidade e eficiência antes de sua aquisição.

\section{CONCLUSÃO}

0 instrumento proposto, apesar de não contemplar todas as etapas necessárias para construção e validação de instrumentos de medida, mostrou-se adequado ao estudo e poderá ser utilizado para orientar os profissionais da CM E na escolha e aquisição de detergentes enzimáticos. Quanto a análise da confiabilidade, pode-se concluir que o instrumento é confiável, segundo o resultado obtido pela análise do alfa de Cronbach, 0,83.

O instrumento construído ficou constituído por 19 itens, dos quais quatro itens foram considerados específicos para avaliação da embalagem, cinco se referem às características do produto e dez estão relacionados a ação do detergente enzimático sobre 0 instrumental.

Quanto à análise dos produtos, por meio da aplicação do instrumento de avaliação, observou-se que o produto P5 obteve a melhor qualidade, média de 78,7 (DP 6,68) e mediana 80,2 e o produto $\mathrm{P} 4$ obteve o pior desempenho, média igual a 56,2 (DP 7,09) e mediana 56,7, com diferença estatisticamente significante neste resultado, sugerindo que a eficiência da limpeza pode depender da qualidade do produto.

Em relação à quantidade e tipo de sujidade, constatou-se que $49,1 \%$ dos instrumentais continham média quantidade de sujidade e 94,5\% apresentavam sangue em sua superfície. Os instrumentais das cirurgias ginecológicas foram os mais utilizados neste estudo (38,2\%).

Os produtos analisados não possuíam as mesmas características, havendo variação na diluição do produto, tempo de contato com o material e número de enzimas. 


\section{REFERÊNCIAS}

1. Silva A. Organização do trabalho na Unidade Centro de Material. Rev Esc Enferm USP. 1998;32(2):169-78.

2.Cruz EA. Novas tecnologias em Centro Cirúrgico e Centro de Material: opinião dos enfermeiros. Rev Baiana Enferm. 1995;8(1/2):102-15.

3. Graziano KU, Balsamo AC, Lopes CLBC, Zotelli M FM, Couto AT, Paschoal MLH. Critérios para avaliação das dificuldades na limpeza de artigos de uso único. Rev Lat Am Enferm. 2006;14(1):70-6.

4. Bergo MCNC, Graziano KU. Validação das máquinas lavadoras desinfectoras automáticas conforme ISO 15.883 e HTM 2030. Rev Enferm UERJ. 2005;13(2):238-44.

5. Cruz EA, Soares E. Prática de enfermagem em Central de Material e Esterilização: uma abordagem estrutural das representações sociais. Rev Enferm UERJ. 2003; 11(2):159-64.

6. Kneedler JA, Darling MH. Using an enzymatic detergent to prerinse instruments. AORN J. 1990;51(5):1326-32.

7. Tlusty FRO. O uso de detergentes enzimáticos na limpeza de artigos médico-cirúrgicos. Rev SOBECC. 1996; 1(1):14-8.

8. Graziano KU. Considerações sobre o uso de detergentes enzimáticos no processo de limpeza: revisão de literatura. Rev SOBECC. 2002;7(4):8-21.
9. Possari JF, Almeida EC. Validação do processo de esterilização com vapor a baixa temperatura e formaldeído (VBTF). Rev SOBECC. 2001;5(5):16-9.

10. Possari JF, Almeida EC. Vapor a baixa temperatura e formaldeído (VBTF): uma nova alternativa para a esterilização de artigos médico-hospitalares. Rev SOBECC. 2000;5(3):25-8.

11. Pinter MG, Gabrielloni MC, Goveia VR, Zecchinato MC, Moura MLPA. Como validar o uso de vapor saturado sob pressão. Rev SOBECC. 1999;4(2):26-30.

12. Baffi SH, Lacerda RA. A busca de qualidade no reprocessamento e na utilização de cateteres de hemodinâmica. Rev SOBECC. 2001;5(6):23-30.

13. Pasquali L. Instrumentos psicológicos: manual prático de elaboração. Brasília: LabPAM; 1999.

14. Stamps PL. Scoring workbook for the Index of Work Satisfaction. M assachusetts: M arket Street Research; 1997.

15. Urbina S. Fundamentos da testagem psicológica. Porto Alegre: Artmed; 2007.

16. Cohen J, Cohen P. Applied multiple regression/correlation for the behavioral sciences. $2^{\text {nd }}$ ed. Hillsdale: New Jersey; 1983.

\section{APÊNDICE 1 - INSTRUMENTO PARAAVALIAÇÃO DE DETERGENTE ENZIMÁTICO}

Este instrumento tem como objetivo principal avaliar a ação de detergentes enzimáticos sobre os instrumentais. Para isso responda as questões a seguir referentes a caracterização do instrumental e do produto.

1. Data: 2. Identificação do produto:

Caracterização do instrumental

1. Tipo de instrumental:

2. Quantidade de sujidade no instrumental

$$
\text { ( )Pequena ( )Média ( )Grande }
$$

3. Tipo de sujidade no instrumental:
( )Sangue
( )Oleosidade
( )Pus
( )Crosta
( )Outros

Caracterização do Produto

1. Parâmetro de diluição:

2. Tempo de contato com o instrumental:

3. Número de enzimas no produto:

continua..

Rev Esc Enferm USP
2008; 42(2):282-9.
www.ee.usp.br/reeuspl


continuação...

4. Apresenta laudo emitido por Laboratório credenciado pelo Ministério da Saúde que comprove a ausência de corrosividade no instrumental?
( ) Sim
( )Não

Responda os próximos 19 itens marcando com um "X" no local que mais corresponde a avaliação do produto. Utilize o quadro abaixo para sua pontuação.

\begin{tabular}{|c|c|c|c|c|}
\hline 1 & 2 & 3 & 4 & 5 \\
\hline Nenhuma/Nada & Pequena parte & Nem muito/nem pouco & Grande parte & Totalmente \\
\hline
\end{tabular}

\begin{tabular}{|c|c|c|c|c|c|}
\hline 1. Provoca irritação à pele e/ou mucosa? & 1 & 2 & 3 & 4 & 5 \\
\hline 2. Houve formação de espuma & 1 & 2 & 3 & 4 & 5 \\
\hline 3. Existem instruções de uso na embalagem? & 1 & 2 & 3 & 4 & 5 \\
\hline 4. As instruções de uso ou modo de usar facilitam a compreensão e a utilização do produto na prática? & 1 & 2 & 3 & 4 & 5 \\
\hline 5. A embalagem é adequada (resistente, com lacre de segurança, fácil de abrir)? & 1 & 2 & 3 & 4 & 5 \\
\hline 6. O produto tem odor agradável? & 1 & 2 & 3 & 4 & 5 \\
\hline 7. O produto é de fácil manuseio, isto é, prático para diluir e usar? & 1 & 2 & 3 & 4 & 5 \\
\hline $\begin{array}{l}\text { 8. O rótulo contém nome do produto, fabricante, lote, validade, registro no Ministério da Saúde e responsável } \\
\text { técnico? }\end{array}$ & 1 & 2 & 3 & 4 & 5 \\
\hline 9. Houve presença de matéria orgânica visível por meio de lupa na parte externa dos artigos após o enxágüe? & 1 & 2 & 3 & 4 & 5 \\
\hline 10. O produto mancha o material? & 1 & 2 & 3 & 4 & 5 \\
\hline 11. O produto mantém o brilho do material? & 1 & 2 & 3 & 4 & 5 \\
\hline 12. Houve oxidação do material? & 1 & 2 & 3 & 4 & 5 \\
\hline 13. Houve presença de matéria orgânica visível a olho nu na parte externa do material após o enxágüe? & 1 & 2 & 3 & 4 & 5 \\
\hline 14. Houve turvação da solução após o primeiro uso? & 1 & 2 & 3 & 4 & 5 \\
\hline 15. Houve eliminação da matéria orgânica do lúmen dos materiais? & 1 & 2 & 3 & 4 & 5 \\
\hline 16. Houve necessidade de escovação após o enxágüe? & 1 & 2 & 3 & 4 & 5 \\
\hline 17. O produto manteve-se eficaz na eliminação de matéria orgânica (saturação)? & 1 & 2 & 3 & 4 & 5 \\
\hline 18. Houve necessidade de relavagem após a avaliação do material a olho nu? & 1 & 2 & 3 & 4 & 5 \\
\hline 19. Houve necessidade de relavagem após avaliação do material por meio de lupa? & 1 & 2 & 3 & 4 & 5 \\
\hline
\end{tabular}

\begin{tabular}{|c|c|c|c|c|c|c|}
\hline \multirow{4}{*}{ 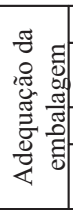 } & 3. Existem instruções de uso na embalagem? & 1 & 2 & 3 & 4 & 5 \\
\hline & $\begin{array}{l}\text { 4. As instruções de uso ou modo de usar facilitam a compreensão e a utilização do produto na } \\
\text { prática? }\end{array}$ & 1 & 2 & 3 & 4 & 5 \\
\hline & 5. A embalagem é adequada (resistente, com lacre de segurança, fácil de abrir)? & 1 & 2 & 3 & 4 & 5 \\
\hline & $\begin{array}{l}\text { 8. O rótulo contém nome do produto, fabricante, lote, validade, registro no Ministério da Saúde e } \\
\text { responsável técnico? }\end{array}$ & 1 & 2 & 3 & 4 & 5 \\
\hline \multirow{5}{*}{ 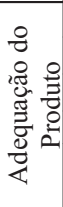 } & 1. Provoca irritação à pele e/ou mucosa? & 1 & 2 & 3 & 4 & 5 \\
\hline & 6. O produto tem odor agradável? & 1 & 2 & 3 & 4 & 5 \\
\hline & 7. O produto é de fácil manuseio, isto é, prático para diluir e usar? & 1 & 2 & 3 & 4 & 5 \\
\hline & 14. Houve turvação da solução após o primeiro uso? & 1 & 2 & 3 & 4 & 5 \\
\hline & $\begin{array}{l}\text { 9. Houve presença de matéria orgânica visível por meio de lupa na parte externa dos artigos após o } \\
\text { enxágüe? }\end{array}$ & 1 & 2 & 3 & 4 & 5 \\
\hline \multirow{10}{*}{ 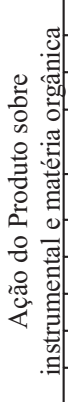 } & 10. O produto mancha o material? & 1 & 2 & 3 & 4 & 5 \\
\hline & 11. O produto mantém o brilho do material? & 1 & 2 & 3 & 4 & 5 \\
\hline & 12. Houve oxidação do material? & 1 & 2 & 3 & 4 & 5 \\
\hline & $\begin{array}{l}\text { 13. Houve presença de matéria orgânica visível a olho nu na parte externa do material após o } \\
\text { enxágüe? }\end{array}$ & 1 & 2 & 3 & 4 & 5 \\
\hline & 2. Houve formação de espuma & 1 & 2 & 3 & 4 & 5 \\
\hline & 15. Houve eliminação da matéria orgânica do lúmen dos materiais? & 1 & 2 & 3 & 4 & 5 \\
\hline & 16. Houve necessidade de escovação após o enxágüe? & 1 & 2 & 3 & 4 & 5 \\
\hline & 17. O produto manteve-se eficaz na eliminação de matéria orgânica (saturação)? & 1 & 2 & 3 & 4 & 5 \\
\hline & 18. Houve necessidade de relavagem após a avaliação do material a olho nu? & 1 & 2 & 3 & 4 & 5 \\
\hline & 19. Houve necessidade de relavagem após avaliação do material por meio de lupa? & 1 & 2 & 3 & 4 & 5 \\
\hline
\end{tabular}

